\title{
GPPS-CH-2020-0091
}

\section{ASSESSMENT OF THE WASHING EFFECTIVENESS OF ON-PURPOSE DESIGNED ECO-FRIENDLY CLEANER AGAINST SOOT DEPOSITS}

\author{
Nicola Casari \\ University of Ferrara \\ nicola.casari@unife.it \\ Ferrara, Italy
}

\author{
Alessandro Vulpio \\ University of Ferrara \\ alessandro.vulpio@unife.it \\ Ferrara, Italy
}

\author{
Michele Pinelli \\ University of Ferrara \\ michele.pinelli@unife.it \\ Ferrara, Italy \\ Craig Appleby \\ ZOK International Group Ltd \\ craig.appleby@zok.com \\ Elsted, Midhurst, West Sussex, UK
}

\author{
Alessio Suman \\ University of Ferrara \\ alessio.suman@unife.it \\ Ferrara, Italy \\ Simon Kyte \\ ZOK International Group Ltd \\ simon.kyte@zok.com \\ Elsted, Midhurst, West Sussex, UK
}

\begin{abstract}
The increment of the industrialization processes led to even more release of carbonaceous particulate into the environment. These airborne contaminants are produced by endothermic machines, coal combustion, heating systems, and production plants. Soot particles suspended into the air can overpass the inlet filters (if present) of gas turbines and deposit onto the internal parts of the compressor. This phenomenon, leading to the modification of the aerodynamic surface of the airfoils, is the main responsible for the gas turbine performance losses over time. This detrimental effect can be partially recovered by washing the compressor unit, frequently.

In this work, the assessment of the washing effectiveness against soot deposits of an on-purpose designed eco-friendly cleaner is provided. The removal effectiveness of this water-based cleaner is related to the capability to collect soot particles from surfaces, limiting redeposit phenomena over the stages. The experimental investigation has been carried out by injecting soot particles, under controlled conditions, into a multistage test axial compressor. Using image post-processing techniques, carried out over the entire compressor flow path, a quantitative evaluation of the washing capability has been assessed. Compared with demineralized water, the cleaner was found to be effective if high cleaning performances are expected.
\end{abstract}

\section{INTRODUCTION}

Atmospheric particle contamination is the main cause of gas turbine compressor fouling. Micrometric particles suspended into the air, after being swallowed by the engine, may adhere to the compressor internal surfaces (Suman et al., 2017). Due to compressor fouling, the efficiency of the gas turbine decreases over time, leading to a reduction of the power output of the whole machine.

The fouling intensity is linked directly to the site location and the engine type. The site in which the engine operates determines the physical and chemical nature of the contaminants: moving from coastal to the desert, or from rural to industrialized areas, the contaminant types can change significantly, and with these, their interaction with the surfaces may be deeply affected. The reinforcement of the interaction between particles and surfaces is strongly influenced by environmental conditions. Due to high humidity levels, water can condense on the surfaces, especially on the first stages of the compressor, increasing the bonding strength between the particles and the substrate (Mezheritsky and Sudarev, 1990). As reported by Stalder (2001), contaminants composed by a mixture of wettable, water-soluble and water-insoluble materials, may become, over time, firmly adhered to the surfaces due to natural aging processes if the deposited layer is not immediately removed. The detrimental effect of fouling can also vary from engine to engine: generally, smaller gas turbines (in terms of output power) show a faster performance decay respect to greater engines in terms of mass flow rate, pressure ratio and efficiency reduction (Tarabrin et al.,1998).

Performance losses due to compressor fouling can be partially mitigated by providing frequent engine cleaning. The online washing technique, widely employed on heavy-duty gas turbines, allows the possibility to recover the engine performance without shutting the machine down. The engine washing is provided by injecting cleaning fluid during the operation using a set of nozzles installed at the inlet bell-mouth of the compressor. To achieve the best results in terms of 
cleaning efficiency and performance recovery, the washing operations are usually provided with a daily frequency (Schneider et al.,2010). Moreover, in stationary applications, the presence of a set of filters, which usually stop contaminants larger than $2 \mu \mathrm{m}$, helps to reduce the fouling rate (Wilcox et al., 2010). Aeronautic engines, due to the absence of filters, are more prone to ingest huge amounts of contaminants, especially in ground operations. As pointed by Asplund (1998), the taxiway before departures, and the use of de-icing products increase the fouling rate of these machines. In the first case, compressor fouling is caused by the ingestion of exhaust gases produced by other engines which operate in idle condition (where the un-burned emission is higher) and dust particles naturally present at low altitudes. In the second case, the deposit build-up is mainly due to the glycol mist injected into the engine. Besides, the absence of a compressor cleaning device mounted permanently on aeronautic engines makes washing operations more challenging to schedule respect to those provided on heavy-duty gas turbines.

The analysis of the type and concentration of the swallowed contaminants, which depend on the specific site location and the operative conditions of the engine, can give an idea of the most appropriate washing regime. In light of this, a lot of attempts and on-field investigations have been made to optimize the washing techniques and to improve the cleaning devices to increase cleaning efficiency and reduce water consumption (Mund and Pilidis, 2006). This on-field research generated, over the years, a significant number of patents that concern washing procedures, cleaning device set-up, nozzle configurations, and design parameters. Despite this, operative parameters such as the droplet diameter and the water-to-air ratio have been highlighted as the most critical aspects to be optimized when washing operations are provided.

Syverud \& Bakken (2007) investigated the effect on the washing effectiveness of different droplet sizes (from $25 \mu \mathrm{m}$ to $200 \mu \mathrm{m})$, and cleaner mass flow rates $(0.4 \%$ to $3.0 \%$ of the water-to-air ratio) during the cleaning operation of a GE J85-13 aero-derivative engine fouled with saltwater. The Authors concluded that higher mass flow rates (up to $3 \%$ of the water-to-air ratio) and coarser droplets (up to $200 \mu \mathrm{m}$ ) represent the most effective combination to achieve the best performance recovery effects and to prevent debris re-deposition in the last stages of the compressor unit. Nevertheless, these parameters, which give the best results in terms of cleaning, are in contrast with customer requests of less water consumption and avoidance of erosion and overall safety issues, which would require low values of the air-to-water ratio and small droplet diameters.

One of the critical parameters for an optimal compressor washing operation is the cleaner fluid. The main characteristic of a cleaner is the presence of surfactant agents, which are usually organic compounds that lower the surface tension of the solution. This mechanism, which leads to the wetting, the penetration, and the dispersion of the deposits, cannot be carried out employing water only. The formation of foam is essential to the degree of effectiveness of the cleaner. The choice of surfactants in an aqueous solution can be used to optimise the elasticity of foam and its resistance to drainage to suit its desired application (Abbott, 2015).

Beyond the cleaning aspect, mandatory aspects for a reliable compressor cleaner are related to human and environmental safety. Commercial cleaners, especially those produced in the early 1980s, contain aromatic hydrocarbon chains that, despite being effective for deposit removal (Kolkmann, 1993), are thought to be dangerous for living species if released into the environment. Stringent requirements were introduced by the Oslo and Paris Commission (OSPAR) in 1998 to prevent the discharge of pollutants or substances harmful for the ecosystem into the North Sea region. This example of progress towards using the most environmentally friendly chemicals for a particular application challenged the compressor cleaning industry to modify the cleaner formulas by removing dangerous compounds, without affecting the cleaning efficiency.

Aim of this work. To quantify the washing effectiveness of a new compressor-cleaner environmentally-safe formula, developed specifically to remove soot deposits from the compressor internal parts, experimental tests have been provided on the multistage test compressor of the Allison $250 \mathrm{C} 18$ engine. The compressor unit has been fouled, by injecting a precise amount of contaminants under controlled conditions, and then washed. The fouled and the washed conditions of the compressor internal parts (rotor blades and stator vanes) have been detected through a photographic report and then compared employing an image-analysis tool. This procedure allowed the quantitative estimation, in terms of residual patterns on the surfaces, of the washing effectiveness of the cleaner. To compare the surface quality resulting from the use of such a cleaner, the analysis has been repeated employing demineralized water, considered in the present study as a reference point.

\section{METHODOLOGY}

In the following section, the methodology used in the present investigation is explained in detail: in the first part, a detailed description of the test facility is provided while the second part is devoted to the explanation of the test conditions, techniques, and materials used in this work. 


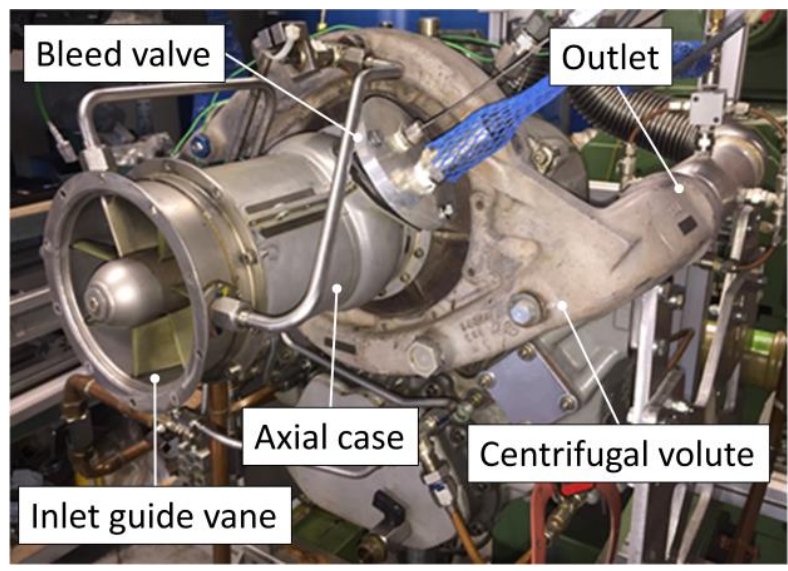

Figure 1 Compressor unit of the Allison 250 C18 engine

\section{Test bench}

The experimental campaign has been carried out on the rotating test facility located in the Fluid Machinery Laboratory of the University of Ferrara. The test facility mounts the multistage test compressor of the Allison $250 \mathrm{C} 18$ engine (Detroit Diesel Allison, 1975) depicted in Fig. 1. The machine has an inlet diameter of $0.104 \mathrm{~m}$, and it is equipped with a fixed inlet guide vane. The compressor has six axial stages and one centrifugal stage. On the axial part, in correspondence of the $5^{\text {th }}$ stage, a bleed valve is located. In the present application, the bleed valve is closed to allow the measurement of pressure and temperature in that zone. The centrifugal stage has two semivolutes, each with a circular exit duct with a diameter of $0.056 \mathrm{~m}$ and two flexible tubes link the outlet ducts to an exhaust outlet with a diameter of $0.100 \mathrm{~m}$. An electric motor drives the machine. At its nominal operative conditions, the compressor achieves a pressure ratio of 6.2 , with a rotational speed of 51,600 rpm and a mass flow rate of $1.36 \mathrm{~kg} / \mathrm{s}$. Besides the compressor unit, an air treatment unit, a water nozzles set-up, a contaminant injection system, and a discharge section compose the test bench. The schematic layout of the test bench is reported in Fig. 2.

The air treatment unit is located at three meters from the compressor inlet mouth. At the inlet of this section is placed a compact EPA F9 filter that avoids the ingestion of external dust without introducing high-pressure losses. The present test bench section aims to modify the relative humidity value of the air swallowed by the compressor using a steam injection nozzle, linked to a steam generator. The steam humidification allows changing the relative humidity of the flow stream without increasing the air temperature.

The water nozzle set-up is composed of four axisymmetric spray cone water nozzles, and it is connected to a volumetric pump and a 501 storage tank thorough a pipeline. A manual control valve downstream of the volumetric pump can regulate the pressure; in the present application, the fluid is supplied towards the nozzle at a pressure of 50 bar. At this pressure value, the calibrated mass flow rate of water injected is $0.18 \mathrm{l} / \mathrm{min}$. The droplets have a Sauter mean diameter of $25 \mu \mathrm{m}$ (values taken from the nozzle manufacturer datasheet). The droplets are injected at a distance of three meters from the compressor inlet mouth: this distance allows the droplets to reach a uniform distribution into the pipe and the kinetic coupling with the airflow.

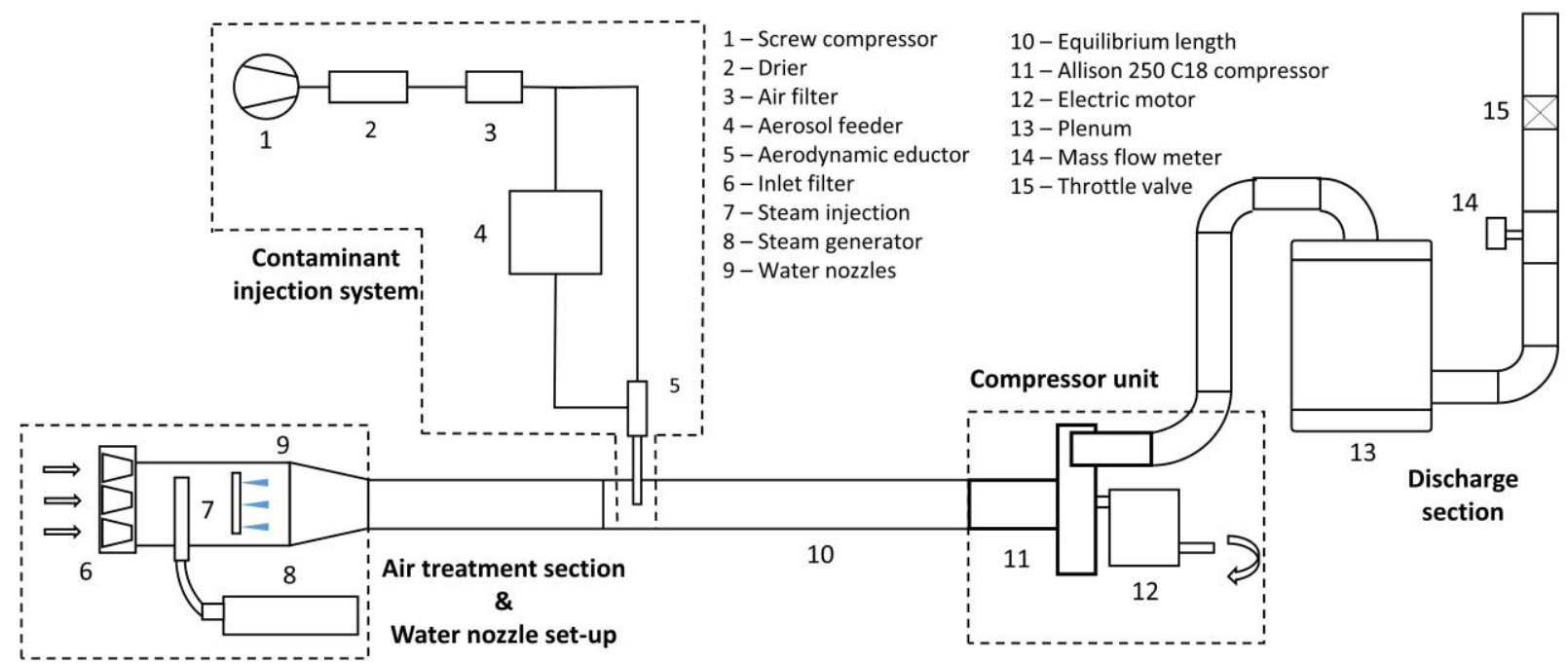

Figure 2 Schematic layout of the test facility 
The contaminant injection system has been designed to supply a constant mass flow rate of dust continuously. A solid aerosol generator doses the powder: the SAG 410 ultra-low flow model made by TOPAS GMBH, which measures out the particle mass flow rate to be injected. The system is powered by a screw compressor equipped with a dryer and a set of filters, which provides dry and clean shop air. The dosed powder is blown by an educator, which deagglomerates and disperses the contaminants into the compressor's main pipeline. The uses of the eductor, which works with the Venturi effect, guarantees an optimal deagglomeration of the test powders thanks to the shear stresses exerted into the throat of the device. The particles are injected at a distance of two meters from the compressor inlet mouth: this distance allows the powder to reach a uniform distribution into the pipe and the kinetic coupling with the airflow.

The downstream section of the compressor is composed of the plenum, a mass flow meter, and a throttling valve.

\section{Test procedure}

Due to the long time required for the deposition phenomena to occur in actual operating conditions, the faithful reproduction of these mechanisms in the laboratory is practically unachievable. Researchers have adopted the strategy to increase the concentration of the contaminants by several orders of magnitude respect to the ambient condition (Jensen et al.,2005) to accelerate the phenomena and realize the test in a laboratory time scale.

In this study, the compressor has been fouled with the standard test dust Raven 410 provided by Powder Technology GMBH. This test dust, commonly known as Carbon Black, is a form of paracrystalline carbon produced by the incomplete combustion of hydrocarbon and heavy petroleum products and is widely present in soot produced by diesel engines (Omidvarborna et al., 2015). In Fig. 3a, the picture of a Carbon Black powder sample is shown, in Fig. 3b, the image of the soot particles taken with the Scanning Electronic Microscope (SEM) is reported. The particle diameter distribution has been provided by the vendor, and it is reported in Fig. 3c.

The compressor unit has been fouled for 30 minutes at an operating rotational speed of 20,000 rpm with an air mass flow rate of $0.38 \mathrm{~kg} / \mathrm{s}$. The particle concentration has been kept constant for all the duration of the test at $12 \mathrm{mg} / \mathrm{m}^{3}$, resulting in a total mass of contaminants injected of $9.8 \mathrm{~g}$. The performance curves of the Allison $250 \mathrm{C} 18$ compressor unit in clean conditions are reported in the work of Munari et al. (2017), while the performance losses caused by continuous ingestion of Carbon Black have been measured on the same compressor unit in a previous work by Vulpio et al. (2020).

During the 30 minutes of operation, the relative humidity of the air at the compressor inlet has been kept constant at $80 \%$ RH. This high humidity value promotes particle deposition and increases the adhesion strength between particles and substrate. This condition can be one of the most burdensome for a cleaner removal action.

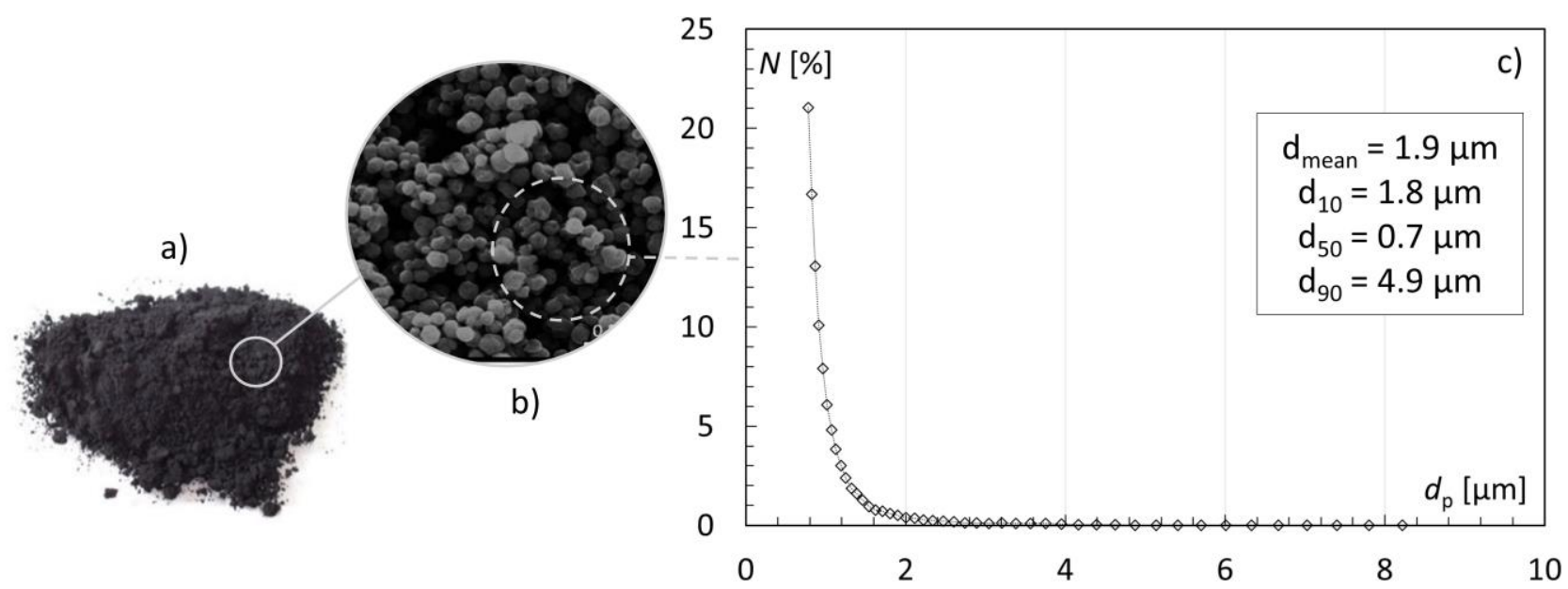

Figure 3 Raven 410 test dust: a) powder sample picture, b) particle SEM picture and c) particle diameter distribution

The washing operation has been divided into two parts, fluid injection ( 1 minute) and drying ( 3 minutes) to eject all the liquid from the compressor. The compressor has been washed with a water-to-air ratio of $1.7 \%$, corresponding to that reported in (Asplund, 1998) for the Allison 250 model.

The novelty introduced by the cleaner tested in this work, respect to other commercial cleaners, relies on a more effective combination of ethoxylated and propoxylated surface-active agents, specifically designed to tackle carbonaceous material particles from metallic surfaces.

To compare the washing effectiveness of the present cleaner with a reference condition, the experimental campaign has been repeated by washing the engine with demineralized water. Before starting the experimental campaign with demineralized water, the compressor has been offline cleaned to restore the initial condition of cleanliness. 
The small size of the Allison $250 \mathrm{C} 18$ compressor, makes this machine easy to be disassembled: the removal of the stator case from the compressor axial part allows direct access to the vanes and blades of the unit. The detection of the fouled and washed zones of the compressor has been done employing dedicated cameras (CANON EOS M6 mirrorless digital camera with Tokina $100 \mathrm{~mm}$ lens with a resolution of 3552 x 2664 pixels) positioned to detect the entire flow path of the axial compressor directly from the lab-testing facility. Thank the disassembling procedure, the half compressor case (stator) was positioned on a realized-on-purpose jig, and it overlooks a proper light and camera set-up. By contrast, the rotor was detected without any disassembling procedure, and the camera and light setup are installed directly on the rotating test rig. The pictures are taken using the same light set-up after each test. In Fig. 4 the pictures of the compressor IGV (Fig. 4.a), rotor wheel (Fig. 4.b), and stator vanes (Fig. 4.c) are reported. The pictures have been taken in clean condition, after the contaminant ingestion, and after the washing procedure. Between each photographic report, the compressor has been dismounted and re-assembled.
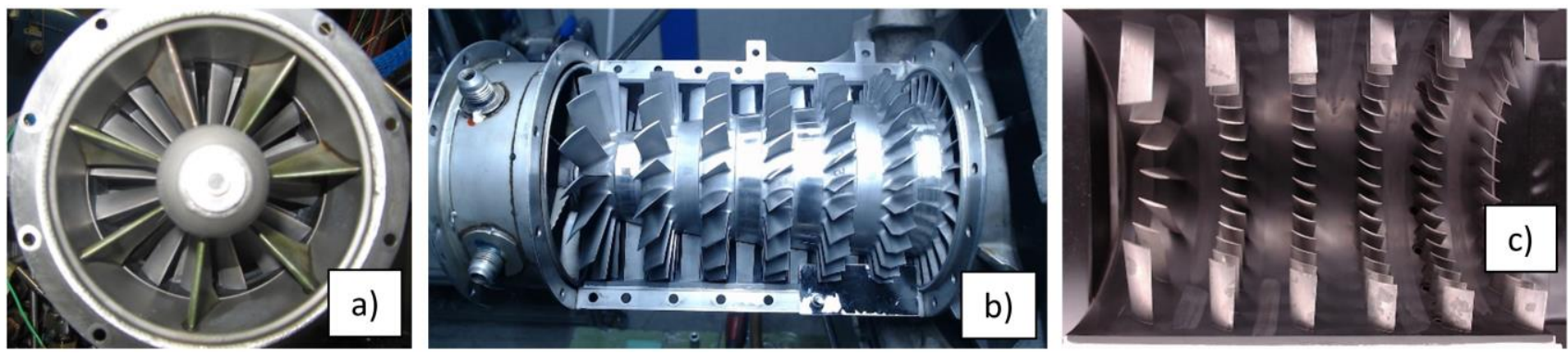

\section{Figure 4 Views of the compressor unit in clean conditions: a) IGV, b) rotor wheel and c) the disassembled stator}

Summing up, the experimental procedure is composed of the following steps:

1) Stator case disassembling and the picture of the IGV, stator vanes and rotor blades (clean condition) are taken;

2) Compressor fouling: 30 min of compressor operation at 20,000 rpm with particles ingestion;

3) Stator case disassembling and the picture of the IGV, stator vanes and rotor blades (fouled condition) are taken;

4) Stator case reassembling and compressor washing: $1 \mathrm{~min}$ of fluid injection at 20,000 rpm;

5) Compressor drying: 3 min of operation at 20,000 rpm;

6) Stator case disassembling and the picture of the IGV, stator vanes and rotor blades (washed condition) are taken.

Starting from the pictures taken during the campaign, the quantification of the washing effectiveness has been carried out by using image processing techniques. The open-source image processing package named IMAGE J (Schneider et al., 2012), developed by the U.S. National Institutes of Health, was used to analyze the digital pictures. Using the same procedure reported in (Brun et al., 2015) and in (Suman et al., 2020), a macro has been used to subtract the picture of the clean stator and rotor to the picture of the dirt one and to convert the resultant image into a binary format. The same procedure has been applied to the clean and after-washed pictures of the rotor and stator. This procedure allows highlighting the dirty zones of the compressor stator and rotor surfaces. The subtraction process applied to the images consists of a pixel-by-pixel subtraction operation: for each pixel pair subtracted, the procedure gives back a white pixel if the result is zero (the two pixels were identical) or a black pixel if the result is non-zero (the pixel were different). In light of this, the subtraction process returns a black and white image, where the black pixels are representative of the dirty zones of the compressor. This procedure can be applied only if the pictures taken at the different conditions are perfectly stackable. The use of a global thresholding value allows choosing a cutoff value, such that every pixel less than that value is considered a white pixel, while every pixel greater than that value is considered a black pixel (Ferreira and Rasband, 2012)

\section{Uncertainty quantification}

The uncertainty evaluation has to be related to the image processing operation of the stator and rotor in clean and fouled conditions. Two different sources of uncertainty have been calculated and combined: i) the component related to the repeatability of the photographic operation $\left(U_{c}\right)$ and ii) the component related to the repeatability of the fouling process $\left(U_{f}\right)$.

The first component, which may be introduced by the electromagnetic noise of the camera and light set-up during the measurements, has been detected through the comparison of the stator and rotor pictures taken in clean conditions (before starting the dust injection in the two tests), according to the procedure explained in the previous section. Similarly, the number of black pixels counted on each vane/blade (due to the differences between two pictures of clean condition), after the subtraction process, compared to the total number of the vane/blade pixel, is representative of the first uncertainty component, named $U_{c}$. Since this uncertainty source is assumed as equally distributed over the measurement range, it is considered to have a rectangular probability distribution. This implies that the present values have to be divided by $3^{1 / 2}$ for 
calculating the standard deviation equivalents. Regarding the second component, its source is found into the intrinsic random motion, which affects dust particles suspended in a medium. Particles with sizes comparable to the mean-free-path of the gas, which is comprised between $0.05 \mu \mathrm{m}$ and $2 \mu \mathrm{m}$, exhibit a random motion resulting from the forces exerted by the gas molecules on them. This condition leads to a statistical process that may influence the deposition patterns found on the vanes/blades. A similar method applied for the $U_{c}$ component quantification is employed to estimate this source of uncertainty: the pictures resulting from the subtraction of the fouled and clean stator/rotor have been subtracted between each other to highlight the different pixels. The ratio between the pixels counted on a vane/blade and the total pixel of the vane/blade gives the estimation of the $U_{f}$ component. Similarly to the previous estimation, this source is divided by $3^{1 / 2}$ for calculating the standard deviation equivalent. Indicating with $i$ the stator/rotor stage, the combination of these uncertainty sources can be calculated as:

$$
\delta U_{i}=\sqrt{\left(\frac{U_{c, i}}{\sqrt{3}}\right)^{2}+\left(\frac{U_{f, i}}{\sqrt{3}}\right)^{2}}
$$

Through the present analysis, an estimation of the expected confidence band associated with the results can be obtained. The uncertainty values will be applied to the results reported in the following section by error bands.

\section{RESULTS}

The results of the application of the image post-processing technique are reported in Figs 5 and 6 for the rotor blades and the stator vanes, respectively. The columns of Figs 5 and 6 refer to the condition of the compressor: the first column shows the fouled blades and vanes after the ingestion of the contaminants, while in the second column the residual deposits after the washing operation are depicted. Instead, the rows of Figs 5 and 6 refer to the cleaning fluid adopted: the first row refers to the demineralized water, while the second row refers to the commercial cleaner.

The post-processed pictures of the rotor blades and stator vanes, obtained after the particle ingestion during the two campaigns (Figs 5a, 5c, 6a, and 6c), show high similarity between them, meaning that the particle deposition operation has good repeatability between the two tests. Both for stator vanes and rotor blades, the pressure sides appear dirtier than the suction sides, especially in the first stages of the compressor. Instead, looking at Figs 5b, 5d, 6b, and 6d, obtained after the washing operations, it can be noted high differences concerning the zones and magnitude of the deposit patterns. From a visual analysis, it can be assessed that the commercial cleaner is able to remove deposits especially in the latter stages of

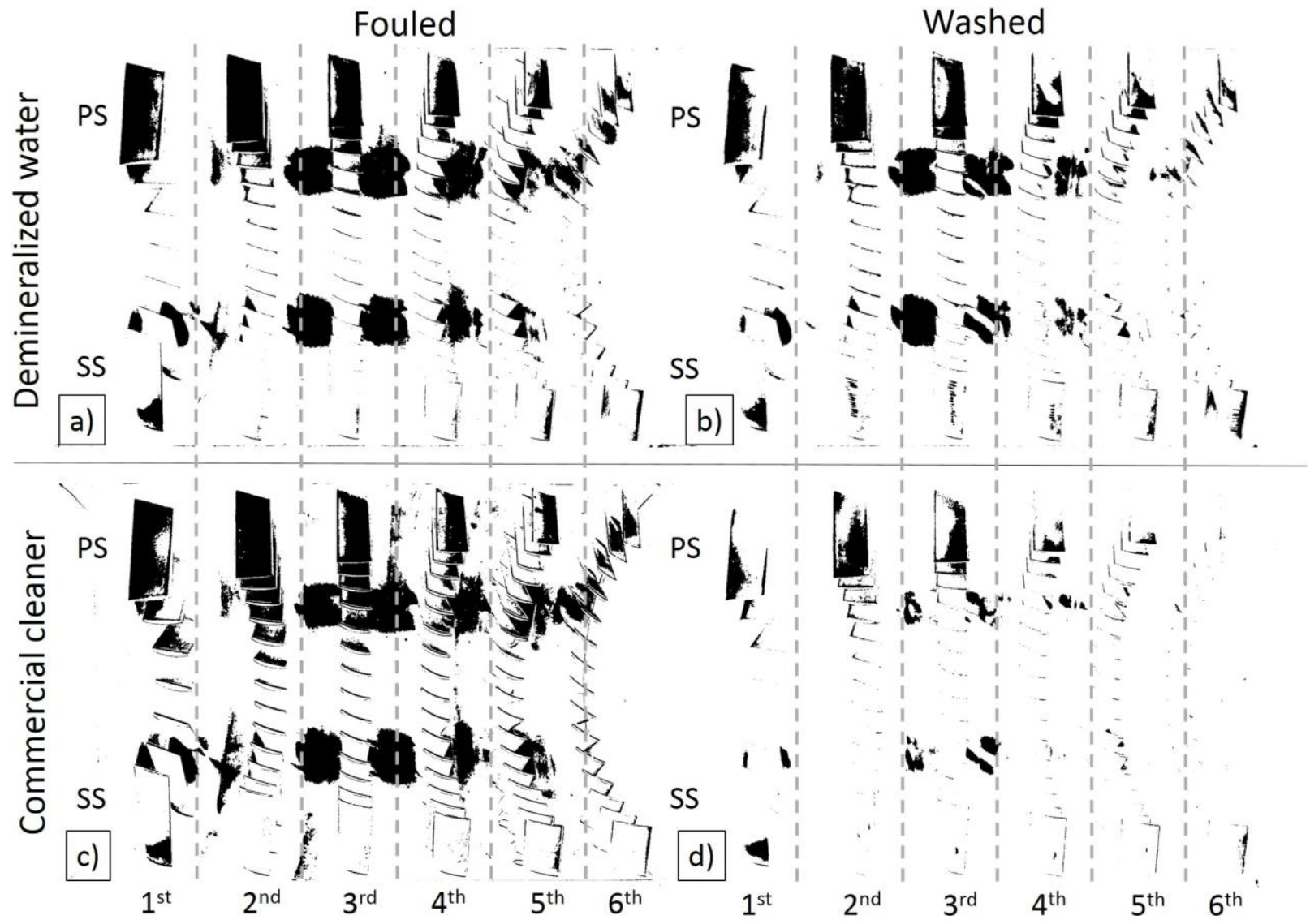

Figure 5 Results of the image post-processing: deposits on the stator vanes after the particle ingestion (a-c) and the washing procedure with demineralized water (b) and commercial cleaner (d). 

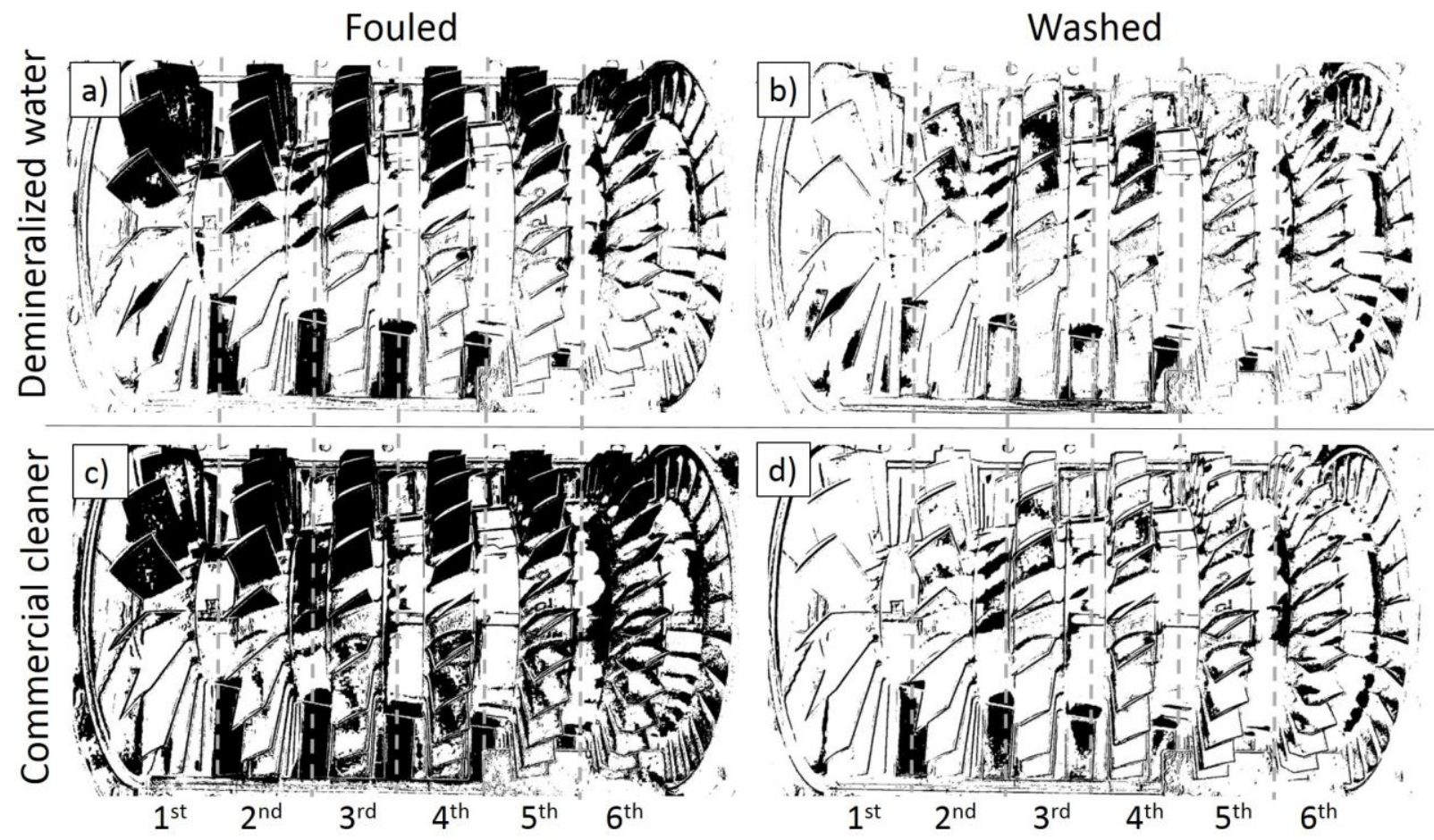

Figure 6 Results of the image post-processing: deposits on the rotor blades after the particle ingestion (a-c) and the washing procedure with demineralized water (b) and commercial cleaner (d).

the compressor, both for rotor and stator. These findings show the different behavior of demineralized water and commercial cleaner in tackling similar deposition patterns when the same washing parameters are employed. The difference in the removal mechanisms of the two cleaner is evident looking at the $2^{\text {nd }}, 3^{\text {rd }}$, and $4^{\text {th }}$ stages of the compressor stator vanes, in correspondence of the suction side. The use of the water instead of the commercial cleaner promotes the motion and the re-deposition of the contaminants from the first stage to the following stages. This behavior is due by the different nature of the two fluids: the presence of surfactant micelles into the commercial cleaner and the formation of the foam promotes the removal and the dispersion of the particles, which continue their path towards the outlet avoiding redeposition.

To extract quantitative information from the post-processed pictures, a macro that counts the pixel on delimitated areas of the pictures has been implemented: the zones taken into account are the blade and vane regions. This technique has been employed to count the black pixels on a vane/blade and to compare them with the vane/blade total number of pixels. This value gives the quantitative information of the surface percentage occupied by the deposits after the washing procedure (residual deposits). This technique has been employed on the picture of the fouled and washed conditions, for both the pressure side and suction side.

The values obtained are employed to compute the washing effectiveness defined as:

$$
\eta_{\text {washing }}=\frac{P_{\text {fouled }}-P_{\text {washed }}}{P_{t}}
$$

where $P_{\text {fouled }}$ and $P_{\text {washed }}$ are the number of the black pixels counted in the fouled and washed post-processed pictures, and $P_{t}$ is the total number of pixels (i.e. the blade or vane surface). The washing capability trends over the stages with the associated error bands are depicted in Fig. 7.

Regarding the trend reported in Fig. 7 for the stator vanes pressure side (Fig. 7a) and for the suction side (Fig. 7b) the commercial cleaner shows higher values of washing capability respect to the demineralized water. The commercial cleaner has an average value of $\eta_{\text {washing }}$ of $47.7 \%$ on the pressure side and $3.4 \%$ on the suction side. The water average values are $18.5 \%$ on the pressure side and $-2.9 \%$ on the suction side.

The effectiveness of this methodology to detect the removal capability is higher for the pressure side, where the amount of deposits is higher. For this reason, the pixel count in the PS is easier and the removal capability can be assessed with the reference of several "fouled" pixels. By contrast, as can be noted by looking at Fig. 5, the suction side is less affected by deposits and the cleaning capability has to be evaluated on a fewer number of "fouled" pixels introducing a higher uncertainty level. The negative values reached by the washing capability values confirm the previous statements: the carbon black deposits, removed from the IGV and the first stage, redeposit onto the following vanes, increasing the vane surface 

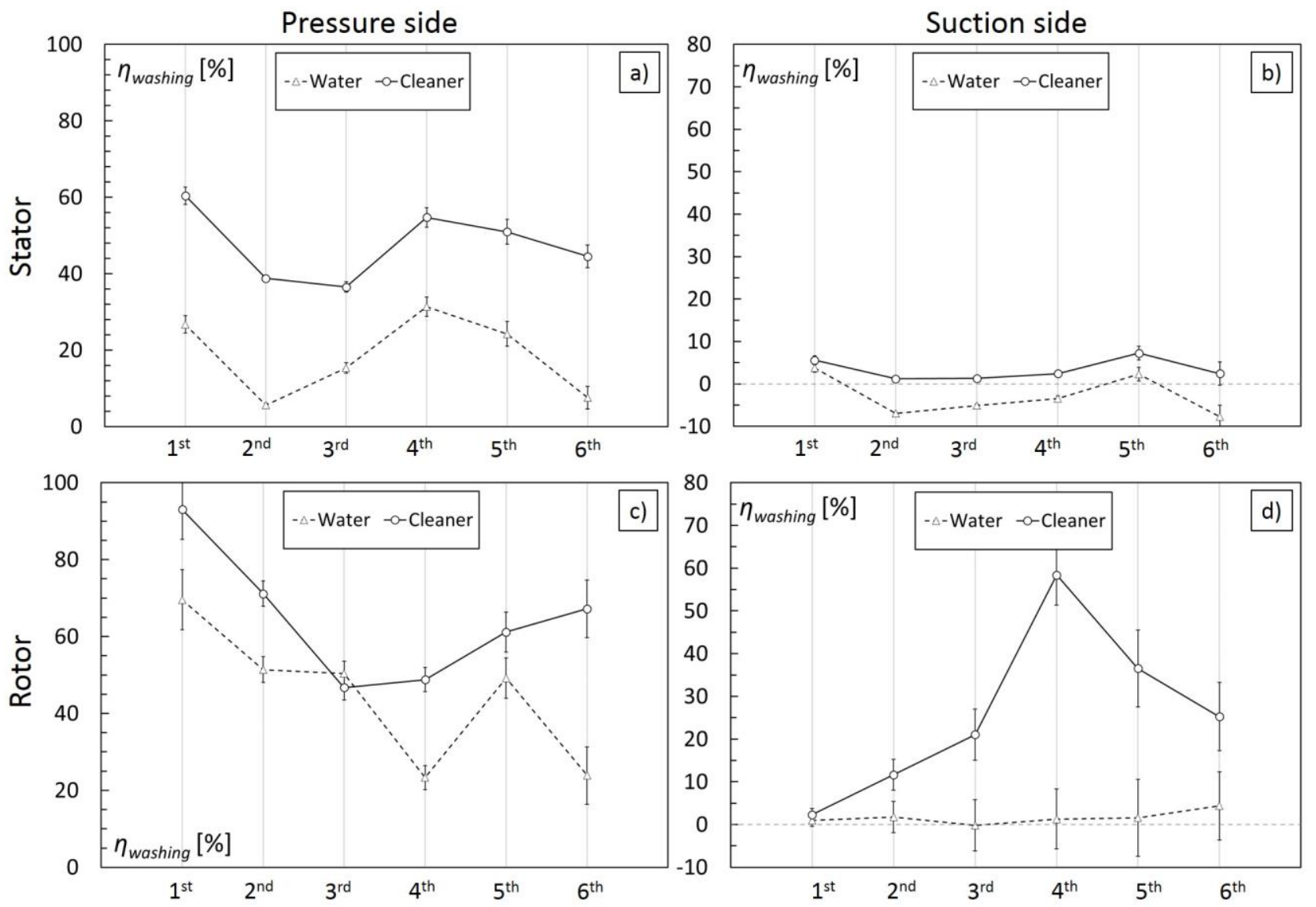

Figure 7 Washing effectiveness and related error bands for the demineralized water (dashed line) and commercial cleaner (solid line) through the stator vanes in correspondence of the pressure side (a) and the suction side (b), and through the rotor blades in correspondence of the pressure side (c) and the suction side (d).

deposit detected at the end of the test. For this reason, a negative value of the washing effectiveness means that, after the washing procedure, the surface is found to be dirtier than the fouled condition.

The same procedure has been applied to the rotor blades, and the results are reported in Fig. 6. Looking at the washing capability trends for the rotor blades depicted in Fig. 7 for the pressure side (Fig. 7a) and the suction side (Fig. 7b) of the blades, it can be noted that, also in this case, the use of the cleaner allows a better washing effectiveness respect to the use of demineralized water only. In particular, as for the stator vanes, the washing capability reaches higher values in correspondence of the pressure side: the average washing capability value along the stages is $44.6 \%$ for the water and 64.7 $\%$ for the commercial cleaner. The pressure side surfaces of the first stages are those with higher washing capability. A very high difference between the washing capabilities of the two fluids is highlighted at the suction side of the rotor blades. In fact, in these zones, the average washing capability is $1.6 \%$ and $25.9 \%$ respectively for water and commercial cleaner. Comparing Figs. $7 \mathrm{a}$ and $7 \mathrm{~b}$ with Fig. $7 \mathrm{c}$ and $7 \mathrm{~d}$, it can be noted that the washing capability values of the rotor blades are higher respect to values found on the stator vanes. This fact probably is due to the centrifugal forces exerted by the rotor that promotes the deposit removal by the mechanical action of the injected droplets.

\section{FURTHER DEVELOPMENTS}

In this work, the use of the picture post-processing technique, applied to the compressor-washing field, proved to be a reliable method to assess the washing effectiveness of a cleaning fluid against contaminants deposited into axial compressors. Further developments will employ this promising technique to investigate the washing effectiveness changing the droplet diameter, the water-to-air ratio, and the type of contaminant.

\section{CONCLUSIONS}

Compressor washing operations have become increasingly attractive due to their capability to recover the performance losses of the engines. The study of the effect of an on-purpose designed compressor cleaner against soot deposits has been assessed in this work. The compressor unit of the Allison $250 \mathrm{C} 18$ engine has been fouled with soot contaminant under controlled conditions and then washed. To compare the results the procedure has been repeated by using demineralized 
water. The compressor has been dismounted and pictures of the fouled zones have been taken and analyzed employing an image post-processing package.

Through the pixel counting technique, the washing capability of the two fluids has been assessed: for the suction sides and the pressure sides of the stator vanes and rotor blades the value of the washing effectiveness has been computed. The results have shown significantly higher values of the washing effectiveness when the commercial cleaner is used, especially on the suction side of the stator vanes and on the pressure side of the rotor blades. Moreover, the re-deposition of soot particles on the suction side of the middle stages of the stator has been detected when demineralized water is used instead of the commercial cleaner.

\section{NOMENCLATURE}

\section{Acronyms}

IGV Inlet guide vane

PS Pressure side

SEM Scanning electron microscope

SS Suction side

\section{Symbols}

d Diameter

N Number distribution

$\mathrm{P} \quad$ Pixel number

U Uncertainty

$\begin{array}{ll}\text { Subscripts } & \\ c & \text { Referred to clean condition (uncertainty) } \\ f & \text { Referred to fouled condition (uncertainty) } \\ \text { fouled } & \text { Referred to fouled condition (pixel counting) } \\ i & \text { Stage index } \\ \mathrm{p} & \text { Particle } \\ t & \text { Total } \\ \text { washed } & \text { Referred to washed condition (pixel counting) } \\ \text { washing } & \text { Washing (pixel counting) }\end{array}$

\section{Greek letters}

$\eta$

Efficiency

\section{REFERENCES}

Abbott, S. J. (2015) Surfactant science: Principles and practice. Destech. Retrieved from: https://www.stevenabbott.co.uk/books. php; https://www.stevenabbott.co.uk/practical-surfactants/thebook.php

Asplund, P. (1998). "Gas turbine cleaning upgrade (compressor wash)". Technical Research Centre of Finland, BALTICA IV: Plant Maintenance for Managing Life \& Performance 1, pp. 105-117.

Brun, K., Grimley, T. A., Foiles, W. C., and Kurz, R. (2015). "Experimental evaluation of the effectiveness of online waterwashing in gas turbine compressors". Journal of Engineering for Gas Turbines and Power, 137(4), p. 042605

Detroit Diesel Allison, Division of General Motors Corporation, Indianapolis, IN 46206 (1975). "Allison gas turbine. operation and maintenance manual. Turboshaft models 250-C18, A, B \& C.”.

Ferreira, T., and W.S. Rasband. (2012). ImageJ user guide. National Institutes of Health, Bethesda, MD. Available at: http://imagej.nih.gov/ij/docs/guide/index.html.

Jensen, J.W., Squire, S.W., Bons, J. P., and Fletcher, T. H. (2005). "Simulated land-based turbine deposits generated in an accelerated deposition facility”. Journal of Turbomachinery, 127(3), pp. 462-470.

Kolkman, H. J. (1993). "Performance of Gas Turbine Compressor Cleaners". Journal of Engineering for Gas Turbines and Power, 115(3): 674-677.

Mezheritsky, A., and Sudarev, A. V. (1990). "The mechanism of fouling and the cleaning technique in application to flow parts of the power generation plant compressors". In ASME 1990 International Gas Turbine and Aeroengine Congress and Exposition, American Society of Mechanical Engineers, pp. V004T11A003-V004T11A003.

Munari, E., Morini, M., Pinelli, M., Spina, P. R., and Suman, A. "Experimental Investigation of Stall and Surge in a Multistage Compressor”. Journal of Engineering for Gas Turbines and Power, 139(2): 022605

Mund, F. C., and Pilidis, P. (2006). "Gas turbine compressor washing: historical developments, trends and main design parameters for online systems". Journal of Engineering for Gas Turbines and Power, 128(2), pp. 344-353. 
Omidvarborna, H., Kumar, A., and Kim, D.-S. (2015). "Recent studies on soot modeling for diesel combustion". Renewable and Sustainable Energy Reviews, 48, pp. 635-647

Schneider, C. A., Rasband,W. S., and Eliceiri, K.W. (2012)."Nih image to imagej: 25 years of image analysis”. Nature methods, 9(7), p. 671.

Schneider, E., Demircioglu Bussjaeger, S., Franco, S., \& Therkorn, D. (2010). “Analysis of compressor online washing to optimize gas turbine power plant performance". Journal of Engineering for Gas Turbines and Power, 132(6) : 062001.

Stalder, J.-P. (2001). “Gas turbine compressor washing state of the art: Field experiences ”. Journal of Engineering for Gas Turbines and Power, 123(2), pp. 363-370.

Suman, A., Morini, M., Aldi, N., Casari, N., Pinelli, M., and Spina, P. R. (2017). “A compressor fouling review based on an historical survey of ASME Turbo Expo papers". Journal of Turbomachinery, 139(4), p. 041005.

Suman, A., Vulpio, A., Casari, N., Pinelli, M., Kurz, R., Brun, K. (2020). "Deposition pattern analysis on a fouled multistage test compressor". Proceedings of ASME Turbo Expo 2020: Turbomachinery Technical Conference and Exposition, Article in press.

Syverud, E., and Bakken, L. E. (2007). “Online Water Wash Tests of GE J85-13”. Journal of Turbomachinery, 129(1): 136-142.

Tarabrin, A., Schurovsky, V., Bodrov, A., and Stalder, J.-P. (1998). “An analysis of axial compressor fouling and a blade cleaning method". Journal of Turbomachinery, 120(2), pp. 256-261.

Vulpio, A., Suman, A., Casari, N., Pinelli, M., Kurz, R., Brun, K. (2020). “Analysis of time-wise compressor fouling phenomenon on a multistage test compressor: performance losses and particle adhesion". Proceedings of ASME Turbo Expo 2020: Turbomachinery Technical Conference and Exposition, Article in press.

Wilcox, M., Baldwin, R., Garcia-Hernandez, A., and Brun, K (2010). "Guideline for gas turbine inlet air filtration systems”. Gas Machinery Research Council, Dallas, TX. 